\title{
Adenoviral Keratoconjunctivitis: Diagnosis, Management, and Prevention
}

\author{
Irene C. Kuo ${ }^{1}$ \\ Published online: 1 May 2019 \\ (C) The Author(s) 2019
}

\begin{abstract}
Purpose of Review This review will provide an updated overview of the diagnosis, management, and prevention of adenoviral keratoconjunctivitis.

Recent Findings Human adenovirus (HAdV) is undergoing nomenclature changes because of whole genome sequencing. Recombination has led to newly identified ocular pathogens like HAdV-D53, HAdV-D54, and HAdV-D56. No quick, highly sensitive and specific diagnostic test is available for ocular adenoviral infections. PCR takes a few hours but is not widely available. Results of using PCR in employees at one medical center support the view of endemic HAdV in the community.

Summary Infection control is highly important as no treatment for ocular HAdV infections exists, ocular sequelae can be significant, and HAdV is endemic. Diagnosis of HAdV ocular infections can be challenging; ophthalmologists are best positioned to diagnose the condition and provide antibiotic stewardship. Development of an accurate diagnostic test with quick turnaround would lead to major benefits in terms of less antibiotic use and fewer work furloughs. Outbreaks occur when infection control becomes lax.
\end{abstract}

Keywords Adenovirus $\cdot$ Epidemic keratoconjunctivitis $\cdot$ Conjunctivitis $\cdot$ Epidemic $\cdot$ Infection control

\section{Introduction}

Viruses cause about $80 \%$ of all cases of acute conjunctivitis [1-6], and human adenoviruses (HAdVs) are believed to account for approximately $65-90 \%$ of cases of viral conjunctivitis [7], making them the most common cause of viral conjunctivitis. Japan and Germany have national monitoring of adenoviral conjunctivitis whereas there is no mandatory reporting in the USA. The risk of infection from household contact has been estimated to be $10-20 \%$, according to an analysis done at the Illinois Eye and Ear Infirmary in Chicago of an outbreak involving close to 100 people [8].

This article is part of the Topical Collection on Ocular Microbiology and Immunology

Irene C. Kuo

ickuo@jhmi.edu

1 Department of Ophthalmology, Wilmer Eye Institute, Johns Hopkins University School of Medicine, 4924 Campbell Blvd \#100, Baltimore, MD 21236, USA
The cost of adenoviral conjunctivitis can be approximated by the lost productivity of the infected individuals who have to be furloughed from work and by the cost of antibiotic drops that are commonly prescribed but do not cure or curtail infection. There are other costs (e.g., family members taking time off from work to care for the patient, cost of visits to the doctor or urgent care or other provider). In addition are less tangible costs such as co-workers taking on additional work when a worker is furloughed for infection. In 2008, an analysis funded by the company producing a point-of-care test for adenoviral conjunctivitis estimated cost savings of $\$ 430$ million using this test [9]; studies in individuals with adenoviral conjunctivitis have not replicated the sensitivity of this test, an issue that will be discussed below [10]. No matter the methodology, the costs of adenoviral conjunctivitis, let alone epidemic keratoconjunctivitis (EKC), are substantial.

Adenoviruses are non-enveloped viruses with an icosahedral protein capsid containing a 34- to 36-kb-long linear, double-stranded DNA genome. HAdVs infect various mucosal membranes, including the ocular surface and respiratory, genitourinary, and gastrointestinal systems [11-14]. Respiratory as well as gastrointestinal infections can be lethal; hence, a 
vaccine against HAdV types 4 and 7 (HAdV-E4, HAdV-B7) was developed for military recruits.

HAdV outbreaks occur globally in communities and in closed or crowded settings like school dormitories, healthcare settings, and housing for military personnel. In fall 2018, about a dozen deaths occurred in long-term pediatric facilities in New Jersey due to adenoviral respiratory infection [15]. Thirty-five students developed adenoviral pneumonia at the University of Maryland in late 2018; one student died [16]. Because of their lipophilic, non-enveloped structure, adenoviruses are highly resistant to commonly used chemical and physical agents, adding to the risk of epidemics. In addition, excreted HAdVs is detectable in stool for weeks to months after infection. The virus may persist in immunosuppression, which may explain why so many adenoviruses identified since the 1980s have been isolated from patients with AIDS. In 2014, the United States Centers for Disease Control and Prevention initiated a voluntary, surveillance registry for adenoviral infections [17]. Although epidemics of adenoviral infection capture the public's attention, most likely HAdVs exist endemically in the community and do not cause severe illnesses for the most part. In this review, we will focus on adenoviral infections of the eye, specifically new developments in diagnosis, infection control, and treatment.

\section{Recombination Gives Rise to New Adenoviral Pathogens; New Technologies Lead to Changes in Nomenclature}

The name "adenovirus" is derived from the fact that the first HAdV (prototype 3) was isolated in 1953 from human adenoids by two independent groups. Because of broad tissue tropisms and ease with which their genomes can be altered, adenoviruses are commonly used in gene therapy.

At least $72 \mathrm{HAdV}$ genotypes with whole genome sequence data are recognized in Genbank [18], divided into 7 species (A-G), with HAdV-D containing the most members and associated with the viruses causing conjunctivitis. The first 51 types were typed serologically (hence, "serotypes"), while types 52 through 72 were typed by whole genome sequencing and phylogenomics [19-21].

Serologic testing is still the most common method of identification. However, the two types of serologic tests- serum neutralization and hemagglutination inhibition-limit our ability to detect new clinical isolates, which adenovirus has a propensity to develop through recombination. The viral capsid consists of three major proteins: the hexon, penton base, and fiber. Serum neutralization tests humoral immunity to the hypervariable loops of the hexon, the most abundant capsid protein. Hemagglutination inhibition identifies the fiber protein. All of the original 51 serotypes are now recognized as genotypes after whole genome sequencing $[18,22]$.
Homologous recombination-documented in adenoviruses in vitro since the 1970s [23-25] — appears to occur only between HAdV types within the same species and in regions of high sequence homology [26, 27]. Recombination gives rise to newly identified adenoviral pathogens, which can lead to human infections not associated with their parental strains.

In addition to causing conjunctivitis and keratoconjunctivitis, HAdV-D genomes recombine more frequently than do other species. HAdV-D genomes continue to evolve via homologous recombination rather than by base substitution in hypervariable coding regions for surface epitopes or immune modulatory proteins $[28 \cdot \bullet]$.

Recombination of the hypervariable regions of the hexon gene is especially important for HAdV-D evolution. The previously serologically named types are undergoing a nomenclature change. For example, HAdV-D19, associated with epidemic keratoconjunctivitis (EKC), has been re-named HAdV-D64 because sole reliance on serum neutralization and/or hexon genotyping led to mistyping [29-31].

Whole genome sequencing, bioinformatics, and detailed in vivo infection analysis are helping to characterize some viruses as the product of recombination of other HAdVs; DNA sequencing might have resulted in a different (and erroneous) genotype. One example is HAdV-D53, which these methods identified as the product of recombination among HAdV-D37, D22, and D8 [32-34]. It turns out that HAdVD53 is a highly contagious corneal pathogen and was the first of three recently identified viruses associated with EKC in the last decade or two-HAdV-D53, D54, and D56 [35]. In December 2018, the United States Centers for Disease Control and Prevention (CDC) reported an outbreak of EKC caused by HAdV-D53 at an optometry clinic in Los Angeles due to lax disinfection measures [36]. While this genotype has caused EKC in Japan and Germany, this CDC report described the first EKC outbreak due to HAdV-D53 in the USA.

In short, newly identified viruses with cornea tropism have been identified in multiple countries; some are already emerging as important pathogens [3]. We cannot tell whether these viruses emerged recently or were only detected recently.

\section{Types of Adenoviral Ocular Infections, Diagnosis, and Antibiotic Stewardship}

The most severe (and dreaded) manifestation of ocular adenoviral infection is EKC; EKC involves both the conjunctiva (bulbar and other surfaces) and corneal epithelium and can cause long-lasting (sometimes permanent) ocular morbidity. The extent of involvement includes corneal subepithelial infiltrates, symblepharon formation, conjunctival membranes, and pseudomembranes, all of which can cause visual disturbance or pain or photophobia. Classically, it has been taught that serotypes 8, 19 (now 64 by whole genome sequencing), 
37, and less frequently 4 are associated with EKC. HAdV-D53 and HAdV-54 are novel types identified as the causative agent in a few recent outbreaks of EKC. In fact, in a 2010 publication from Japan, the three most common types causing EKC in descending order were types 37,54 , and 53 , whereas serotype 8 was becoming less common in Japan over two decades [37].

The authors examined relative proportions of causative types of EKC by (re-)typing conjunctival swabs of patients from six countries (Japan, USA, Saudi Arabia, Nepal, Vietnam, Bangladesh, and Austria) between 2000 and 2009 [38]. They found great disparity in the HAdV types. Whereas HAdV-D8 had greatly decreased from being the leading cause of EKC in Japan in the early 1990s, it was still a major cause of EKC in the five other countries. In those countries, HAdVD53 and HAdV-D54 were not common causes of EKC in this time period, although they were common types in Japan. The authors raised the possibility that cases thought to have been caused by HAdV-D8 before newer diagnostic technology may have actually been caused by HAdV-D54 $[38,39]$. It is not possible to identify HAdV-D54 using serum neutralization testing because of an absence of serotype-specific antiserum. HAdV-D54 has been isolated from samples in Japan dating back to 1995 but was only identified in 2006-2008 [33, 34]. Because of similarities to type 8 , it is believed that HAdV-54 evolved from HAdV-8 [37].

In a 6-year study at a tertiary eye clinic, one fourth of patients with adenoviral keratoconjunctivitis had recurrent or persistent symptoms caused by subepithelial infiltrates (SEI; also known as "nummuli") lasting more than 45 days [40] (Fig. 1). These SEI, which occur at approximately day 10 , can give rise to irregular astigmatism, scatter light, and result in photophobia. Moreover, one fourth of patients developed membranes or pseudomembranes during acute infection, and one out of seven of this subset developed symblepharon. These results "belie the commonly held view that adenovirus keratitis is invariably of limited duration . . and suggest a significant level of longterm morbidity" [40] A less severe manifestation of ocular adenoviral infection is pharyngoconjunctival fever (PCF), associated with types 3, 5, 7, and 11 [7]. Acute hemorrhage conjunctivitis (AHC) is often considered a subset of EKC with significant subconjunctival hemorrhages.

Given EKC has the most striking findings and is not usually a diagnostic dilemma, the bigger diagnostic challenge is the less severe types of adenoviral follicular conjunctivitis caused by probable endemic viral types in the community. The clinical course of adenoviral conjunctivitis varies from inapparent infection to severe conjunctivitis, and HAdV can be easily transmitted. Although HAdV was not found in normal asymptomatic control cases in a study from Japan (implying it is not normal conjunctival flora) [41] or in fellow eyes during the incubation period in cases of bilateral sequential involvement [42], the authors found fellow patients and the

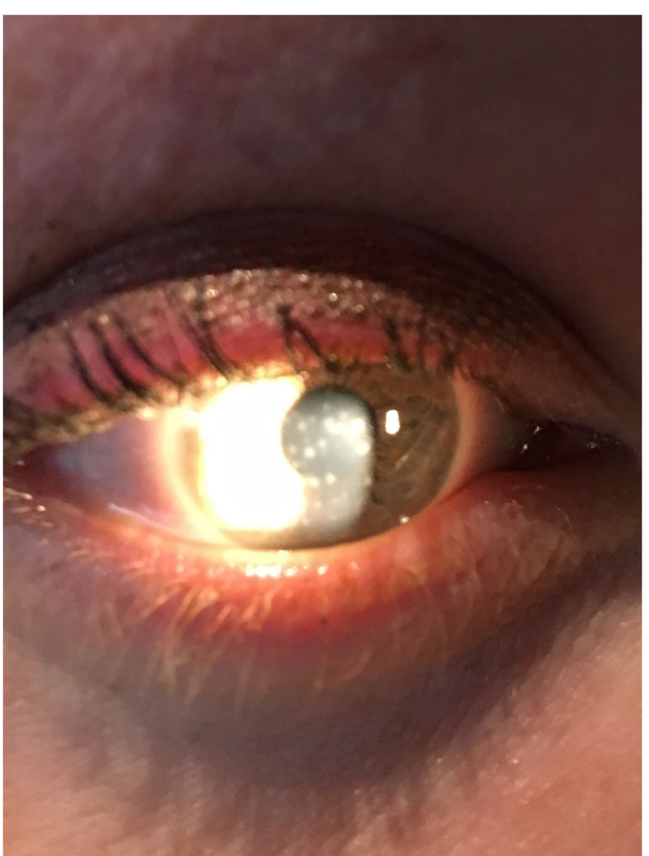

Fig. 1 Unilateral subepithelial infiltrates that developed after an acute episode of epidemic keratoconjunctivitis. They persisted despite a month of four times daily prednisolone acetate $1 \%$

ophthalmologist on the ward had mild or inapparent infection with the same HAdV type as an inpatient with EKC [41]. The authors posit that mild/inapparent infections may play a role in community or hospital-acquired HAdV-conjunctivitis.

Adenoviral conjunctivitis can be difficult to differentiate from other causes of acute conjunctivitis. As clinical diagnosis is imperfect, viral cell culture and PCR have been the gold standards [43], but these methods are being usurped by whole genome sequencing. An unpublished report discussed in a review paper found that a clinical roundtable of experts in external ocular diseases had a clinical accuracy slightly below $50 \%$ in diagnosing adenoviral conjunctivitis [7]. The main reason is the considerable overlap in signs and symptoms between viral and bacterial disease. In addition, there is no universally accepted, highly sensitive, highly specific diagnostic test with quick turnaround. Culture results for adenovirus may take weeks. Polymerase chain reaction is the other gold standard but is not widely in use outside academic centers; at a few centers like the University of Pittsburgh Charles T. Campbell Eye Microbiology Lab (http://eyemicrobiology. upmc.com/Adenovirus.htm) and at Johns Hopkins Hospital, PCR for adenovirus has been validated for conjunctival adenovirus. The test can be ordered for patients and employees at these two institutions; because of expense and essential laboratory expertise, however, PCR is not widely used. Shell vial culture (taking a few days) and Adenoclone are far less common diagnostic methods. A second-generation point-of-care diagnostic immunoassay that can detect the virus hexon antigen is available [44]. The result of the AdenoPlus 
(Quidel, San Diego, CA) returns quickly; though specificity is high, sensitivity can range widely [45, 46]. As such, AdenoPlus may be better used to diagnose patients with adenoviral conjunctivitis than to screen patients with possible adenoviral conjunctivitis.

Specifically, depending on publication and location, when compared with PCR, AdenoPlus has a sensitivity of 39.5$90 \%$ and specificity of approximately $96 \%$ [45, 46]. Reasons for the range of sensitivity values may be different swabbing techniques or different profiles of endemic adenoviral serotypes depending on geographical area. Although the AdenoPlus labeling states that the test is best used within 7 days of developing a red eye consistent with infectious conjunctivitis, this instruction was not heeded in these studies. The instructions for use also state, "Negative results do not preclude ... infection nor are they intended to rule out other microbial-caused infections of the conjunctiva, and should not be used as the sole basis for treatment or other management decisions." Whereas labeling of the first generation test AdenoDetector (with lower sensitivity than the AdenoPlus) [47] was required by the United States Food and Drug Administration to specify that any negative test needed to be followed up by culture or PCR [48], that labeling was removed for the AdenoPlus.

Despite the improved sensitivity of AdenoPlus, it is wise to obtain definitive testing if the patient is highly suspicious for adenoviral infection yet has a negative AdenoPlus result. Because of a potentially false negative rate $(22 \%$ in one study from the UK) and because the positive predictive value is affected by prevalence, some authors conclude that AdenoPlus "should. . . be used only selectively in patients in whom there is a diagnostic suspicion and a high pretest probability for adenoviral conjunctivitis." [46] In addition, the test was cleared by the FDA for use in adults, not in children, but children may account for many cases of adenoviral conjunctivitis because of hygiene issues. Therefore, the sensitivity and specificity of AdenoPlus are not known in the pediatric population. A positive AdenoPlus in adults may be useful because of its high specificity, but a negative result should lead the provider to do further testing like PCR or viral culture and/ or refer to an ophthalmologist who would have more clinical acumen than the other provider types who see conjunctivitis.

Because of training and experience seeing eye disease and pathology, ophthalmologists are still the best equipped to diagnose patients with adenoviral conjunctivitis. The clinical diagnostic accuracy of non-ophthalmologists is likely lower than the accuracy of ophthalmologists. However, most patients with acute conjunctivitis do not see ophthalmologists or optometrists. In a recent study of enrollees in a large US managed care network, more than $80 \%$ of patients with acute conjunctivitis were diagnosed by healthcare providers other than ophthalmologists and optometrists [49••]. These providers include family practice physicians, internists, urgent care physicians, pediatricians, and non-doctors (i.e., nurse practitioners and physician assistants in primacy care and at urgent care facilities). The proportion of persons with newly diagnosed acute conjunctivitis who filled prescriptions for topical antibiotics within 14 days of their initial diagnosis was lowest for patients seen by ophthalmologists compared to patients seen by other types of providers [49••]. Nearly $60 \%$ of enrollees in this managed care network filled antibiotic prescriptions for acute conjunctivitis; the highest antibiotic fill fraction was for patients seen by urgent care providers. In other words, patients seen by ophthalmologists had the lowest odds of filling antibiotic prescriptions. This finding has many ramifications. Antibiotic stewardship is important to decrease the risk of resistance to antibiotics that do not cure or alter the course of adenoviral conjunctivitis. Second, proper referral to an ophthalmologist and proper antibiotic stewardship will likely result in less cost to the healthcare system and patients because of less antibiotic use and less chance of unfortunate side effects from antibiotics.

Interestingly, ocular adenoviral infection is not just limited to the conjunctiva and cornea. Orbital/brain imaging (done either incidentally or because the extent of lid and facial swelling with adenoviral conjunctivitis can resemble orbital cellulitis) has revealed that infection induces an inflammatory process that extends deep into the orbit [50]. Imaging demonstrates edema and inflammation of the periocular tissue in the anterior orbit, enlargement of the lacrimal gland, and nasolacrimal duct compromise. These findings suggest that adenovirus conjunctivitis is accompanied by dacryoadenitis and dacryocystitis.

\section{Transmission and Prevention (Infection Control) in Healthcare Settings; Description of a Triage Algorithm Used at a Major Academic Center}

Transmission is through contact with ocular secretions. Infected persons are probably contagious before symptoms arise. With a breach in infection control, outbreaks can occur especially as the virus is resistant to typical disinfectants like $70 \%$ isopropyl alcohol and 3\% hydrogen peroxide; desiccated, viable virus can be recovered after 5-7 weeks on fomites [51]. Therefore, infection can spread through contact with instruments like tonometers, lid specula, dropper bottles, and slit lamps, not all of which come in disposable form; respiratory and ocular outbreaks have occurred in a neonatal intensive care unit because of contaminated lid specula and other equipment (some not even contacting the patient) used to examine infants for retinopathy of prematurity $[52,53]$.

To add to the risk of transmission, infected individuals tend to rub or wipe their eyes because of discomfort and discharge in the affected eye. One paper described how adenovirus 
could be cultured from the hands of almost $50 \%$ of individuals with EKC in an ophthalmology clinic [54]. Since adenoviruses are non-enveloped and are hydrophilic, alcohol-base hand sanitizers are not effective; soap and water hand washing are the better choice for removal [55••]. Aside from pieces of ophthalmic equipment that contact the ocular surface of patients during a typical examination, common surfaces like chair armrests, doorknobs, and counter surfaces that an infected individual contacts can potentially harbor the virus. These surfaces should be cleaned thoroughly after a potentially infected patient is seen.

In ophthalmology, methods of cleaning tonometry applanation tips have evolved over time with the advent or discovery of new infectious agents (e.g., prions, human immunodeficiency virus), but a recent Ophthalmic Technology Assessment by the American Academy of Ophthalmology concludes 1:10 dilute bleach (sodium hypochlorite) is a single high-level disinfectant with broad efficacy against common infectious agents encountered in eye care [56]. Only 1:10 dilute bleach is recommended by both the tonometer manufacturers and the CDC for disinfecting applanation tonometers. Outbreaks of EKC can be quite large [57-60] and have a high financial cost [61].

If there is suspicion of infection, isolate the patient of interest in an exam room as soon as possible to avoid being in the waiting room in contact with other patients and fomites common to all (Table 1) $[60,62,63]$. While examining the patient, use disposable tonometer tips and single-use eye drops (discard any multi-dose eye drop containers that may have been used on the patient). After the visit concludes, disinfect the slit lamp (handles, chin rest, forehead rest, etc.) for an adequate amount of time; also disinfect other surfaces the patient may have contacted including arm rests, countertops, pens, and door knobs. Environmental Protection Agency (EPA)-registered intermediate level disinfectants that are bactericidal, tuberculocidal, and virucidal are commercially available. The recommended contact time with a pre-moistened wipe containing quaternary ammonium and isopropyl alcohol

Table 1 Control of epidemic keratoconjunctivitis

Management of an outbreak of EKC in a healthcare setting [60, 62, 63]

Ensure all hospital employees have been educated about the policy

Early recognition by trained personnel

Hand hygiene and gloving between encounters with suspect patients or employees

Limit number of entrances to clinical area where outbreak is occurring and institute triage at those entrances

Isolate affected individuals ("red eye" room(s), where drops are either single use or discarded at day's end; no applanation tonometry; wipe all surfaces with germicide after each patient)

Work furlough for affected employees is $2 \mathrm{~min}$, then allowing to air-dry. These infection control practices were stressed after an investigation of an optometry clinic where an EKC outbreak involving 17 patients occurred [36].

Dating to a few near-outbreaks of EKC in the Department of Ophthalmology at Johns Hopkins Hospital, an isolation room had been set up in the ophthalmology department's eye emergency room for ophthalmology residents to screen "red eye" patients and potentially culture and furlough these employees [64].

Administrative changes led to closure of both the red eye room and Wilmer Emergency Room, and hence, the need for an updated "red eye" policy. This policy was the product of discussions among Hospital Epidemiology and Infection Control, the Occupational Health Division, the Virology division, and the chief of infection control for the Department of Ophthalmology (author ICK).

In 2011, Johns Hopkins Medicine institutionalized a new "red eye" policy. The focus was to triage employees with potential adenoviral conjunctivitis in a swift manner, diagnose infection accurately and relatively quickly, and furlough infected employees, thus preventing transmission to patients and other employees $[65,66]$. Accurate diagnosis was a highlight of this new policy because clinical diagnosis has limited accuracy and might lead to (unnecessary) furloughs of noninfected employees. Having a healthy workforce is vital to the operations of any work area, particularly in healthcare where understaffing could affect patient outcomes and the risk of nosocomial infections must be minimized.

Because the top concerns were employee health and safety and preventing spread to patients and other employees, the "red eye" algorithm focused on employees although a triage algorithm was also developed a call center to use for nonemployees. We have continued to monitor outcomes of this policy, a Johns Hopkins Medicine quality improvement initiative, in terms of employees with (1) "red eye" of any etiology, (2) PCR-positive adenoviral conjunctivitis, and (3) EKC. This triage algorithm consists of swift triage by nurse practitioners in the Occupational Health division who were trained by a cornea specialist (author ICK) to swab the conjunctivae of employees suspected of having adenoviral conjunctivitis. Employee swabs are sent for PCR of the hexon region, developed and validated for conjunctival specimens by the Pathology department of Johns Hopkins Hospital. There is no commercially available PCR test for the detection of adenovirus in conjunctival specimens.

PCR results (whether "positive" or "negative") return within a few hours; typing takes a few days. During this time, PCR-positive employees remain furloughed from work. The results of typing help tailor the discussion and expectations of work furlough. PCR-positive employees with types associated with EKC are told they will be off work for a minimum of 2 weeks from onset of symptoms. PCR-positive employees 
without EKC types are told they will be off work for a minimum of 1 week from symptom onset. Nurse practitioners stress that these are estimated times to resolution. In all cases, employees must return to Occupational Health for screening and clearance before they return to duty. The nurse practitioners contact the Ophthalmology department for questionable or refractory cases.

Although the importance of triage, accurate diagnosis, and furlough become evident in hindsight after an outbreak of EKC in any location, most likely large medical centers can more easily adopt a PCR-based algorithm with the support of their virology laboratories. Centers considering a similar algorithm will have to develop tests that utilize non-commercial primers or adapt tests cleared for use with nasopharyngeal samples. The success of such an algorithm also relies on having a centralized site, e.g., Occupational Health, manage the triage, diagnostic testing, and work furloughs, rather than letting employees scatter to various professionals who may not recognize the condition as being highly contagious and whose diagnostic acumen is surpassed by PCR [66]. Whole genome sequencing will likely become cheaper and more accessible as well.

Five-year results of the Johns Hopkins Medicine quality improvement effort showed that relatively consistently, the proportion of employees who were swabbed by nurse practitioners for suspicion of adenoviral conjunctivitis and turned out to be PCR-positive remained 7-10\% when data were analyzed at 21 months [65], 36 months [66], and 60 months [55••]. The proportion of PCR-positive employees who had EKC also remained approximately $25 \%$ in these time periods. Interestingly, genotypes responsible for EKC changed over 5 years [55••]. Because of lack of clustering and work places of affected employees, most cases of adenoviral conjunctivitis in JHM employees are believed to be community-acquired, and this change in type found in hospital employees may reflect change in endemic community types. This finding is consistent with international literature. In Japan, (which like Germany, has national monitoring of adenoviral conjunctivitis), it is believed that types causing EKC occur with baseline prevalence in the community and the types change over time [67]. Endemic types may also vary by geographical region [68].

Another finding from the 5-year analysis of Johns Hopkins Medicine employees was that certain work areas have higher rates of adenoviral conjunctivitis than others [55••]. In fact, 10 of 30 work areas accounted for more than $50 \%$ of cases. Outpatient pharmacy, surgery, internal medicine, and ophthalmology had the highest numbers of cases of adenoviral conjunctivitis; rounding out the top 10 work areas were Food Services, Environmental Services, and Security. This finding is similar to findings from a surveillance program in Beijing where food service workers and laborers were overrepresented among patients with adenoviral conjunctivitis. The reason for the overrepresentation of food service employees in both analyses may be lack of proper education regarding hygiene and transmission [69].

Without an historic or concurrent control group, we cannot prove but it is possible that our policy has limited transmission of adenoviral conjunctivitis or EKC at our medical center [55••]. By reducing the number of unnecessary furloughs (furloughs that might have occurred with only clinical diagnosis), employees who are not infected are able to continue to work. In the event of an outbreak, patient care can be affected when more employees than necessary become furloughed.

\section{Treatments}

There has been no effective antiviral drug against HAdVs. The mainstay of EKC management includes accurate diagnosis and supportive, symptomatic treatment, with artificial teardrops. Infrequently, a topical antibiotic is prescribed to prevent or treat bacterial superinfection, which is rare [70, 71].

Povidone-iodine $2 \%$ was used in a pilot study four times a day for a week and led to recovery in three quarters of eyes [72]. A fixed combination of povidone-iodine and dexamethasone for $\mathrm{EKC}$ is in clinical trials. Results of a multi-center, randomized double-masked phase II of povidone-iodine $0.6 \%$ and dexamethasone $0.1 \%$ (PVP-I/dexamethasone) vs. povidone-iodine $0.6 \%$ vs. vehicle $(1: 1: 1)$ used in adults in India with adenoviral conjunctivitis were just reported [73]; several authors (including the senior/corresponding author) were employees of the company manufacturing the PVP-I/ dexamethasone combination. Endpoints like negative cell culture immunofluorescence assay, reduction in watery conjunctival discharge, and decreased bulbar conjunctival redness were utilized and recorded at various time points. PVP-I/dexamethasone was better than vehicle in clinical resolution at day 5 and viral eradication as measured by cell culture as early at day 5 (with evidence of eradication even at day 3), but PVP-I/ dexamethasone was not better than PVP-I at these time points. The study also had endpoints of "global clinical score" and "expanded clinical cure" which were summation and composite scores of watery discharge and bulbar conjunctival redness, for which PVP-I performed better than PVP-I. Because of the geographical differences in endemic types and changes in endemic types in any one location, Phase III results are eagerly awaited as the study expands to six continents and also includes children.

Until studies on the combination antiseptic/corticosteroid are complete, there is no commonly accepted, effective treatment for adenoviral conjunctivitis or sequelae like SEI. Local treatment with steroid eye drops nearly always succeeds in the short term, but often leads to recurrence of SEI once they are stopped [74] and to steroid dependence with prolonged virus persistence in a rabbit model [75]. In addition are the usual 
steroid complications, such as intraocular hypertension and cataract formation.

Cidofovir $1 \%$ was once thought to be promising, but was not shown to alter disease course and caused conjunctival and eyelid toxicity and discomfort $[76,77]$. In one study where cidofovir was used with and without cyclosporine, cidofovir had no effect on the course of infection [78].

Cyclosporine A (CsA) alone has been used for SEI [79]. CsA $1 \%$ was used in 12 eyes of 9 patients who experienced a decrease in the number of medications before and after treatment from $1.88 \pm 1.05$ to $1.22 \pm 0.44$, respectively $(P=$ $0.049)$. Six patients $(66 \%)$ showed clinical improvement, and $3(34 \%)$ were stable during the treatment period. Although there was no change in best-corrected visual acuity, patients reported statistically significant reduction in the severity of symptoms before and after the treatment. Most of the patients reported no foreign body sensation, glare, or other side effects with topical CsA treatment. Overall, patients noted an improvement in vision and satisfaction with CsA $1 \%$ treatment. Tapering/weaning of CsA was not discussed.

The same authors who tried cidofovir with and without cyclosporine reported use of CsA alone in a large series of 70 eyes of 48 patients with SEI, and CsA was found to be effective in eliminating or reducing the SEI in 40 of the eyes without recurrences after discontinuation of the CsA [80]. Sixteen eyes demonstrated no change, and four eyes could not tolerate the symptom of burning from the CsA eye drops. In another study, the majority of patients with longstanding established SEI were successfully treated with $2 \%$ CsA with reduction or elimination of SEI and successful weaning off therapy.

Jeng et al. showed that the use of CsA $1 \%$ drops may be useful in treating patients with subepithelial infiltrates after adenoviral keratoconjunctivitis [81]. Twelve eyes of seven patients had symptomatic SEI develop after adenoviral keratoconjunctivitis that were responsive to corticosteroid eye drops but were resistant to tapering. After the initiation of CsA eye drops, the corticosteroid eye drops could be tapered, and all eyes could be maintained on CsA eye drops once per day or less. Mean follow-up time was 13.0 months (range, 4 28 months). Four of the six eyes that had decreased vision on initial presentation gained best corrected visual acuity after treatment. However, no patient could be tapered off CsA; in each instance, the SEI recurred, necessitating the reinstitution of CsA $1 \%$ four times daily. For each patient, the authors eventually found the lowest effective dose of CsA to maintain the corneas without SEI.

One percent CsA administered 4 times a day for 21 days during the acute infection produced earlier subjective improvement of local symptoms and a trend toward a lower incidence of corneal opacities in a small study with 10 patients in each of 4 arms [82], but the authors recommended further investigation in a greater number of patients [83]. In a study with 3 arms of 30-50 patients each - lubricants, topical prednisolone acetate $1 \%$, or CsA $2 \%$ - authors suggest SEI may be less severe in symptoms and findings in patients receiving topical corticosteroids or topical CsA when compared with patients using only topical lubricants. This conclusion was based on a lower proportion of patients on CsA $2 \%$ having SEI at day 21 compared with the proportion of patients using topical lubricants or steroid drops. The difference was statistically significant. There was no difference at day 7 . The authors also had a clinical scoring system for SEI ("0" for no SEI, "1" for "few" $(\leq 10)$, or " 2 " for "many" $(>10))$ that appeared lower for CsA $2 \%$ patients at day 21 than for steroid or lubricant patients, but no statistical testing was reported. The authors state these results imply that patients with SEI taking CsA 2\% may have a shorter duration of symptoms and findings although 22 patients from the CsA and topical steroid groups required treatment for persistent SEI beyond the 3-week study period. A dozen patients required topical steroids, and 10 needed topical CsA 2\%; there was no mention of their outcome. Although the authors state these patients were followed for 3 months, it is not clear whether they required these medications for the duration or whether they were able to be weaned off CsA or steroid drops.

Topical tacrolimus has also been used for SEI [84]. With a median of about a year of follow-up, in about $60 \%$ of eyes, tacrolimus drops or ointment for a median time of 6 months reduced the number and size of SEI, and in $31.76 \%$, SEI were eliminated. The improvement in visual acuity after treatment was statistically significant. About $19 \%$ of eyes had recurrence after discontinuation for a mean of 7 months.

\section{Conclusion}

In conclusion, adenoviral conjunctivitis can be a major issue as it affects healthcare workers and patients alike, with ophthalmology clinics being especially vulnerable. Epidemic keratoconjunctivitis can lead to chronic morbidity including visual disturbance, corneal scarring, symblephara, and dry eye syndrome. Old nomenclature is being shed as whole genome sequencing confirms surprising amounts of recombination first noted in the 1970s. This feature of HAdV-D species (which includes HAdV types causing EKC) raises the specter of new pathogens for the ocular surface; HAdV-D53, HAdVD54, and HAdV-D56 were only discovered in the last 20 years and cause EKC.

Serologic typing is no longer sufficient. PCR and culture have been gold standards for academic centers, but PCR for adenoviral conjunctivitis is in limited use because of cost and equipment, which are issues for whole genome sequencing as well. Because adenoviral conjunctivitis (EKC in particular) can have such high economic cost—employee furloughs, the 
requirements of affected children and adults to stay home, prescriptions for antibiotics that will not help the infectiondevelopment of accurate diagnostic testing that is affordable and quick is important. Treatment remains mostly supportive although new drugs like fixed combination of povidoneiodine and dexamethasone for EKC are in clinical trials.

One of the most dreaded complications of EKC, subepithelial infiltrates, can be helped by steroid drops, but long-term, refractory cases may require a steroid-sparing agent like cyclosporine. A randomized clinical trial comparing cyclosporine vs. placebo has not yet been performed and could better define the natural history and effect of treatment of this disorder.

\section{Compliance with Ethical Standards}

Conflict of Interest Irene C. Kuo declares that she has no conflict of interests.

Human and Animal Rights and Informed Consent This article does not contain any studies with human or animal subjects performed by the author.

Open Access This article is distributed under the terms of the Creative Commons Attribution 4.0 International License (http:// creativecommons.org/licenses/by/4.0/), which permits unrestricted use, distribution, and reproduction in any medium, provided you give appropriate credit to the original author(s) and the source, provide a link to the Creative Commons license, and indicate if changes were made.

\section{References}

Papers of particular interest, published recently, have been highlighted as:

•• Of major importance

1. Stenson S, Newman R, Fedukowicz H. Laboratory studies in acute conjunctivitis. Arch Ophthalmol. 1982;100(8):1275-7.

2. Rönnerstam R, Persson K, Hansson H, Renmarker K. Prevalence of chlamydial eye infection in patients attending an eye clinic, a VD clinic, and in healthy persons. Br J Ophthalmol. 1985;69(5):385-8.

3. Uchio E, Takeuchi S, Itoh N, Matsuura N, Ohno S, Aoki K. Clinical and epidemiological features of acute follicular conjunctivitis with special reference to that caused by herpes simplex virus type 1 . Br J Ophthalmol. 2000;84(9):968-72.

4. Woodland RM, Darougar S, Thaker U, Cornell L, Siddique M, Wania $\mathrm{J}$, et al. Causes of conjunctivitis and keratoconjunctivitis in Karachi, Pakistan. Trans R Soc Trop Med Hyg. 1992;86(3):317-20.

5. Fitch CP, Rapoza PA, Owens S, Murillo-Lopez F, Johnson RA, Quinn TC, et al. Epidemiology and diagnosis of acute conjunctivitis at an inner-city hospital. Ophthalmology. 1989;96(8):1215-20.

6. Matsui K, Shimizu H, Yoshida A, Nagaoka E, Nishio O, Okuda K. Monitoring of adenovirus from conjunctival scrapings in Japan during 2005-2006. J Med Virol. 2008;80:997-1003.

7. O'Brien TP, Jeng BH, McDonald M, Raizman MB. Acute conjunctivitis: truth and misconceptions. Curr Med Res Opin. 2009;25(8): 1953-61.
8. Vastine DW, West CE, Yamashiroya H, Smith R, Saxtan DD, Gieser DI, et al. Simultaneous nosocomial and community outbreak of epidemic keratoconjunctivitis with types 8 and 19 adenovirus. Trans Sect Ophthalmol Am Acad Ophthalmol Otolaryngol. 1976;81(5):826-40.

9. Udeh BL, Schneider JE, Ohsfeldt RL. Cost effectiveness of a pointof-care test for adenoviral conjunctivitis. Am J Med Sci. 2008;336(3): 254-64. https://doi.org/10.1097/MAJ.0b013e3181637417.

10. Smith AF, Waycaster C. Estimate of the direct and indirect annual cost of bacterial conjunctivitis in the United States. BMC Ophthalmol. 2009;9:13.

11. Arnold J, Jonaska M, Kajon AE, Metzgar D, et al. Genomic characterization of human adenovirus 36 , a putative obesity agent. Virus Res. 2010;149:152-61.

12. Chu W, Pavan-Langston D. Ocular surface manifestations of the major viruses. Int Ophthalmol Clin. 1979;19:135-67.

13. Dingle JH, Langmuir AD. Epidemiology of acute, respiratory disease in military recruits. Am Rev Respir Dis. 1968;97(Suppl):1-65.

14. Wood DJ. Adenovirus gastroenteririts. Br Med J (Clin Res Ed). 1988;296:229-30.

15. Adenovirus. https://nj.gov/health/cd/topics/adenovirus.shtml. Accessed November 17, 2018.

16. Tzacik C. Five more adenovirus cases confirmed at University of Maryland, bringing total to 35 . https://www.baltimoresun.com/ news/maryland/education/higher-ed/bs-md-adenovirus-20181212story.html. Accessed December 17, 2018.

17. Binder AM, Biggs HM, Haynes AK, Chommanard C, Lu X, Erdman DD, et al. Human adenovirus surveillance-United States, 2003-2016. Morb Mortal Wkly Rep. 2017;66:1039-42.

18. Singh G, Zhou X, Lee JY, Yousuf MA, Ramke M, Ismail AM, et al. Recombination of the epsilon determinant and corneal tropism: human adenovirus species D types 15, 29, 56, and 69. Virology. 2015;485:452-9.

19. Liu EB, Ferreyra L, Fischer SL, Pavan JV, Nates SV, Hudson NR, et al. Genetic analysis of a novel human adenovirus with a serologically unique hexon and a recombinant fiber gene. PLoS One. 2011;6:e24491.

20. Liu EB, Wadford DA, Seto J, Vu M, Hudson NR, Thrasher L, et al. Computational and serologic analysis of novel and known viruses in species human adenovirus D in which serology and genomics do not correlate. PLoS One. 2012;7:e33212.

21. Walsh MP, Chintakuntlawar A, Robinson CM, Madisch I, Harrach B, Hudson NR, et al. Evidence of molecular evolution driven by recombination events influencing tropism in a novel human adenovirus that causes epidemic keratoconjunctivitis. PLoSOne. 2009;4(6):e5635.

22. Robinson CM, Seto D, Jones MS, Dyer DW, Chodosh J. Molecular evolution of human species D adenoviruses. Infect Genet Evol. 2011;11:1208-17.

23. Takemori N. Genetic studies with tumorigenic adenoviruses. 3. Recombination in adenovirus type 12. Virology. 1972;47:157-67.

24. Williams JF, Ustacelebi S. Complementation and recombination with temperature-sensitive mutants of adenovirus type 5. J Gen Virol. 1971;13:345-8.

25. Williams JF, Gharpure M, Ustacelebi S, McDonald S. Isolation of temperature-sensitive mutants of adenovirus type 5. J Gen Virol. 1971;11:95-101.

26. Boursnell ME, Mautner V. Recombination in adenovirus: crossover sites in intertypic recombinants are located in regions of homology. Virology. 1981;112:198-209.

27. Williams J, Young H, Austin P. Complementation of human adenovirus type 5 ts mutants by human adenovirus type 12. J Virol. 1975; $15: 675-8$.

28.• Robinson CM, Singh G, Lee JY, Dehghan S, et al. Molecular evolution of human adenoviruses. Sci Rep. 2013;3:1812 Discusses in detail the high rate of recombination in HAdV and how this 
feature is leading to emergence of highly virulent adenoviruses with new tissue tropisms.

29. Walsh MP, Seto J, Jones MS, Chodosh J, Xu W, Seto D. Computational analysis identifies human adenovirus type 55 as a re-emergent acute respiratory disease pathogen. J Virol. 2012;86(8):4693-5.

30. Singh G, Robinson CM, Dehghan S, Schmidt T, Seto D, Jones MS, et al. Overreliance on the hexon gene, leading to misclassification of human adenoviruses. J Clin Microbiol. 2010;48(3):991-3.

31. Zhou X, Robinson CM, Rajaiya J, Dehghan S, Seto D, Jones MS, et al. Analysis of human adenovirus type 19 associated with epidemic keratoconjunctivitis and its reclassification as adenovirus type 64. Invest Ophthalmol Vis Sci. 2012;53(6):2804-11.

32. Walsh MP, Chintakuntlawar A, Robinson CM, Madisch I, Harrach B, Hudson NR, et al. Evidence of molecular evolution driven by recombination events influencing tropism in a novel human adenovirus that causes epidemic keratoconjunctivis. PLoS One. 2009;4(6):e5635.

33. Aoki K, Ishiko H, Konno T, Shimada Y, Hayashi A, Kaneko H, et al. Epidemic keratoconjunctivitis due to the novel hexonchimeric-intermediate 22,37/H8 human adenovirus. J Clin Microbiol. 2008:46:3259-69.

34. Engelmann I, Madisch I, Pommer H, Heim A. An outbreak of epidemic keratoconjunctivitis caused by a new intermediate adenovirus $22 / \mathrm{H} 8$ identified by molecular typing. Clin Infect Dis. 2006;43:e64-6.

35. Kaneko H, Aoki K, Ohno S, Ishiko H. Complete genome analysis of a novel intertypic recombinant human adenovirus causing epidemic keratoconjunctivitis. J Clin Microbiol. 2011;49:484-90.

36. OYong K, Killerby M, Pan CY, Huynh T, Green NM, Wadford DA, et al. Outbreak of epidemic keratoconjunctivitis caused by human adenovirus type D53 in an eye care clinic - Los Angeles County, 2017. MMWR Morb Mortal Wkly Rep. 2018;67(48):1347-9.

37. Kaneko H, Suzutani T, Aoki K, Kitaichi N, Ishida S, Ishiko H, et al. Epidemiological and virological features of epidemic keratoconjunctivitis due to new human adenovirus type 54 in Japan. Br J Ophthalmol. 2011;95(1):32-6.

38. Kaneko H, Iida T, Ishiko H, et al. Analysis of the complete genome sequence of epidemic keratoconjunctivitis-related human adenovirus type 8, 19, 37 and a novel serotype. J Gen Virol. 2009;90: 1471e6.

39. Ishiko H, Shimada Y, Konno T, Hayashi A, Ohguchi T, Tagawa Y, et al. A novel human adenovirus causing nosocomial infections of epidemic keratoconjunctivitis. J Clin Microbiol. 2008;46:2002-8.

40. Butt AL, Chodosh J. Adenoviral keratoconjunctivitis in a tertiary care eye clinic. Cornea. 2006;25(2):199-202.

41. Kaneko H, Maruko I, Iida T, Ohguchi T, Aoki K, Ohno S, et al. The possibility of human adenovirus detection from the conjunctiva in asymptomatic cases during nosocomial infection. Cornea. 2008;27(5):527-30.

42. Kimura R, Migita H, Kadonosono K, Uchio E. Is it possible to detect the presence of adenovirus in conjunctiva before the onset of conjunctivitis? Acta Ophthalmol. 2009;87:44-7.

43. Thompson PP, Kowalski RP. A 13-year retrospective review of polymerase chain reaction testing for infectiousagents from ocular samples. Ophthalmology. 2011;118(7):1449-53.

44. Sambursky R, Trattler W, Shachar T, Starr C. Sensitivity and specificity of the AdenoPlus test for diagnosing adenoviral conjunctivitis. JAMA Ophthalmol. 2013;131(1):17-22.

45. Kam KY, Ong HS, Bunce C, Ogunbowale L, Verma S. Sensitivity and specificity of the AdenoPlus point-of-care system in detecting adenovirus in conjunctivitis patients at an ophthalmic emergency department: a diagnostic accuracy study. Br J Ophthalmol. 2015;99(9):1186-9.
46. Sachdev A, Boukouvala S, Ahluwalia H, Crossman R, Mehta P. Role of the Adenoplus test in refractory, recurrent and clinically undiagnosed conjunctivitis. Can J Ophthalmol. 2018;53(5):529-32.

47. Sambursky R, Tauber S, Schirra F, Kozich K, Davidson R, Cohen EJ. The RPS adeno detector for diagnosing adenoviral conjunctivitis. Ophthalmology. 2006;113(10):1758-64.

48. Siamak NM, Kowalski RP, Thompson PP, Romanowski EG, Shanks RM, Gordon YJ. RPS adeno detector. Ophthalmology. 2009;116(3):591-1.

49.• Shekhawat NS, Shtein RM, Blachley TS, Stein JD. Antibiotic prescription fills for acute conjunctivitis among enrollees in a large United States managed care network. Ophthalmology. 2017;124(8):1099-107 Data show that most patients with conjunctivitis do not see ophthalmologists and there is a need for antibiotic stewardship.

50. Horton JC, Miller S. Magnetic resonance imaging in epidemic adenoviral keratoconjunctivitis. JAMA Ophthalmol. 2015;133(8): $960-1$.

51. Gordon YJ, Gordon RY, Romanowski E, Araullo-Cruz TP. Prolonged recovery of desiccated adenoviral serotypes 5, 8, and 19 from plastic and metal surfaces in vitro. Ophthalmology. 1993;100:1835-9 discussion 1839-1840.

52. Calkavur S, Olukman O, Ozturk AT, Kilic FK, Gulfidan G, Devrim I, et al. Epidemic adenoviral keratoconjunctivitis possibly related to ophthalmological procedures in a neonatal intensive care unit: lessons from an outbreak. Ophthalmic Epidemiol. 2012;19(6):371-9.

53. Sammons JS, Graf EH, Townsend S, Hoegg CL, Smathers SA, Coffin SE, et al. Outbreak of adenovirus in a neonatal intensive care unit: critical importance of equipment cleaning during inpatient ophthalmologic examinations. Ophthalmology. 2019;126(1):13743.

54. Azar MJ, Dhaliwal DK, Bower KS, Kowalski RP, Gordon YJ. Possible consequences of shaking hands with your patients with epidemic keratoconjunctivitis. Am J Ophthalmol. 1996;121(6): 711-2.

55.• Kuo IC, Espinosa C. Five-year trends in adenoviral conjunctivitis in employees of one medical center. Infect Control Hosp Epidemiol. 2018;39(9):1080-5 Employees of a large medical enterprise are screened, swabbed, and furloughed pending accurate diagnosis using PCR. The virus type causing EKC has changed over time. Employees without direct patient contact but in patient care areas can get adenoviral conjunctivitis in higher numbers than expected, speaking to the importance of widespread emphasis on hand hygiene and education.

56. Junk AK, Chen PP, Lin SC, Nouri-Mahdavi K, Radhakrishnan S, Singh K, et al. Disinfection of tonometers: a report by the American Academy of Ophthalmology. Ophthalmology. 2017;124(12):186775 .

57. Hamada N, Gotoh K, Hara K, Iwahashi J, Imamura Y, Nakamura S, et al. Nosocomial outbreak of epidemic keratoconjunctivitis accompanying environmental contamination with adenoviruses. J Hosp Infect. 2008;68(3):262-8.

58. Montessori V, Scharf S, Holland S, Werker DH, Roberts FJ, Bryce E. Epidemic keratoconjunctivitis outbreak at a tertiary referral eye care clinic. Am J Infect Control. 1998;26(4):399-405.

59. Adenovirus-associated epidemic keratoconjunctivitis outbreaksfour states, 2008-2010. MMWR. 2013;62:637-41.

60. Warren D, Nelson KE, Farrar JA, Hurwitz E, Hierholzer J, Ford E, et al. A large outbreak of epidemic keratoconjunctivitis: problems in controlling nosocomial spread. J Infect Dis. 1989;160(6):938-43.

61. Piednoir E, Bureau-Chalot F, Merle C, Gotzmanis A, Wulbout J, Bajolet O. Direct costs associated with a nosocomial outbreak of adenoviral conjunctivitis infection in a long-term care institution. Am J Infect Control. 2002;30(7):407-10.

62. Gottsch JD. Surveillance and control of epidemic keratoconjunctivitis. Trans Am Ophthalmol Soc. 1996;94:539-87. 
63. Kuo IC. More than meets the eye: adenoviral conjunctivitis in healthcare settings. Infect Control Hosp Epidemiol. 2017 Nov;38(11):1358-60.

64. Gottsch JD, Froggatt JW 3rd, Smith DM, et al. Prevention and control of epidemic keratoconjunctivitis in a teaching eye institute. Ophthalmic Epidemiol. 1999;6:29-39.

65. Kuo IC, Espinosa C, Forman M, Pehar M, Maragakis LL, Valsamakis A. Detection and prevalence of adenoviral conjunctivitis among hospital employees using real-time polymerase chain reaction as an infection prevention tool. Infect Control Hosp Epidemiol. 2014;35(6):728-31.

66. Kuo IC, Espinosa C, Michael Forman M, Valsamakis A. A polymerase chain reaction-based algorithm to detect and prevent transmission of adenoviral conjunctivitis in hospital employees. Am J Ophthalmol. 2016;163:38-44.

67. Hiroi S, Morikawa S, Takahashi K, Komano J, Kase T. Molecular epidemiology of human adenoviruses $\mathrm{d}$ associated with epidemic keratoconjunctivitis in Osaka, Japan, 2001-2010. Jpn J Infect Dis. 2013;66(5):436-8.

68. Aoki K, Tagawa Y. A twenty-one year surveillance of adenoviral conjunctivitis in Sapporo, Japan. Int Ophthalmol Clin. 2002;42(1): 49-54.

69. Li J, Lu X, Jiang B, Du Y, Yang Y, Qian H, et al. Adenovirusassociated acute conjunctivitis in Beijing, China, 2011-2013. BMC Infect Dis. 2018;18(1):135.

70. Gigliotti F, Williams WT, Hayden FG, Hendley JO, Benjamin J, Dickens M, et al. Etiology of acute conjunctivitis in children. J Pediatr. 1981;98:531-6.

71. Watanabe Y, Uchio E, Itoh N, Usui N, Nakagawa H, Inoue K, et al. Bacterial infection in the conjunctiva of patients with adenoviral conjunctivitis. Jpn J Ophthalmol. 2001;45:115.

72. Trinavarat A, Atchaneeyasakul LO. Treatment of epidemic keratoconjunctivitis with $2 \%$ povidone-iodine: a pilot study. J Ocul Pharmacol Ther. 2012;28:53-8.

73. Pepose JS, Ahuja A, Liu W, Narvekar A, Haque R. Randomized, controlled, phase 2 trial of povidone-iodine/dexamethasone ophthalmic suspension for treatment of adenoviral conjunctivitis. Am J Ophthalmol. 2018;194:7-15.

74. Romanowski EG, Roba LA, Wiley L, Araullo-Cruz T, Gordon YJ. The effects of corticosteroids of adenoviral replication. Arch Ophthalmol. 1996;114(5):581-5.
75. Laibson PR, Dhiri S, Oconer J, Ortolan G. Corneal infiltrates in epidemic keratoconjunctivitis. Response to double-blind corticosteroid therapy. Arch Ophthalmol. 1970;84(1):36-40.

76. Gordon YJ, Romanowski EG, Araullo-Cruz T. Topical HPMPC inhibits adenovirus type 5 in the New Zealand rabbit ocular replication model. Invest Ophthalmol Vis Sci. 1994;35:4135-43.

77. Gordon YJ, Naesens L, DeClercq E, Maudgal PC, Veckeneer M. Treatment of adenoviral conjunctivitis with topical cidofovir. Cornea. 1996; 15:546.

78. Hillenkamp J, Reinhard T, Ross RS, Böhringer D, Cartsburg O, Roggendorf M, et al. The effects of cidofovir $1 \%$ with and without cyclosporin a $1 \%$ as a topical treatment of acute adenoviral keratoconjunctivitis: a controlled clinical pilot study. Ophthalmology. 2002;109:845-50.

79. Levinger E, Slomovic A, Sansanayudh W, Bahar I, Slomovic AR. Topical treatment with $1 \%$ cyclosporine for subepithelial infiltrates secondary to adenoviral keratoconjunctivitis. Cornea. 2010;29(6): 638-40.

80. Reinhard T, Godehardt E, Pfahl HG, Sundmacher R. Local cyclosporin A in nummuli after keratoconjunctivitis epidemica. A pilot study. Ophthalmologe. 2000;97:764-8.

81. Jeng BH, Holsclaw DS. Cyclosporine A $1 \%$ eye drops for the treatment of subepithelial infiltrates after adenoviral keratoconjunctivitis. Cornea. 2011;30(9):958-61.

82. Hillenkamp J, Reinhard T, Ross RS, Böhringer D, Cartsburg O, Roggendorf M, et al. Topical treatment of acute adenoviral keratoconjunctivitis with $0.2 \%$ cidofovir and $1 \%$ cyclosporine: a controlled clinical pilot study. Arch Ophthalmol. 2001;119(10):148791.

83. Asena L, Şıngar Özdemir E, Burcu A, Ercan E, Çolak M, Altınörs DD. Comparison of clinical outcome with different treatment regimens in acute adenoviral keratoconjunctivitis. Eye (Lond). 2017;31(5):781-7.

84. Berisa Prado S, Riestra Ayora AC, Lisa Fernández C, Chacón Rodríguez M, Merayo-Lloves J, Alfonso Sánchez JF. Topical tacrolimus for corneal subepithelial infiltrates secondary to adenoviral deratoconjunctivitis. Cornea. 2017;36(9):1102-5.

Publisher's Note Springer Nature remains neutral with regard to jurisdictional claims in published maps and institutional affiliations. 